\title{
Identifying risk factors for Plasmodium infection and anaemia in Kinshasa, Democratic Republic of Congo
}

Giovanfrancesco Ferrari ${ }^{1,2^{*}}$ (1), Henry M. T. Ntuku ${ }^{1,2,3}$, Amanda Ross ${ }^{1,2}$, Sandro Schmidlin ${ }^{1,2}$, Didier M. Kalemwa ${ }^{1,2}$, Antoinette K. Tshefu ${ }^{3}$ and Christian Lengeler ${ }^{1,2}$

\begin{abstract}
Background: There is little data on the risk factors for malaria infection in large cities in central Africa and in all age groups. There may be different associations with the risk factors for areas with different malaria transmission intensities such as the effect of fever or age. This study aimed at identifying risk factors associated with Plasmodium infection and anaemia among children 6-59 months and individuals aged older than 5 years in Kinshasa, a large city with heterogeneity in malaria prevalence.
\end{abstract}

Methods: This study analysed data from 3342 children aged 6-59 months from 25 non-rural health zones (HZs) and for 816 individuals aged older than 5 years from two HZs in Kinshasa (non-rural), collected during a cross sectional malaria survey in 2011. Logistic regression with random effects was used to investigate predictors for malaria and anaemia. Differences in risk factors in areas with a prevalence of less than 10 and $10 \%$ or greater were investigated.

Results: There was evidence of a different age-pattern in the two transmission settings. For children under 5 years, the highest prevalence of malaria was observed in the 48-59 months group in both transmission settings, but it increased more gently for the lower transmission $\mathrm{HZs}(p=0.009)$. In a separate analysis in children over 5 years in two selected $\mathrm{HZs}$, the peak prevalence was in 5-9 years old in the higher transmission setting and in 15-19 years old in the lower transmission setting. Reported fever was associated with malaria in both transmission strata, with no evidence of a difference in these associations $(p=0.71)$; however in children older than 5 years there was a significant interaction with a stronger association in the low transmission $\mathrm{HZ}$. Insecticide-treated net (ITN) use was associated with a lower risk of malaria infection in children 6-59 months in the high transmission HZs. Similar estimates were found in children over 5 years and the lower transmission $\mathrm{HZ}$ but the associations there were not significant. There was no evidence of a difference in these associations by strata. The risk of anaemia decreased with increasing age in all strata, whereas it increased with malaria infection and reported fever. ITN use did not show evidence of protection against anaemia. Low socio-economic status was associated with malaria in high transmission setting in children 6-59 months and anaemia in low transmission setting.

Conclusions: This study shows that in areas of low transmission in Kinshasa, the peak prevalence occurs in older age groups however ITN use was highest in children under 5 years. Targeted distribution of ITN to all age groups should be continued. For most risk factors, there was no evidence of an interaction with transmission intensity however the associations with age and with fever in the last 2 weeks did vary significantly.

Keywords: Malaria, Malaria risk, Anaemia, Fever, ITN use, ITN ownership, Democratic Republic of Congo, Kinshasa

\footnotetext{
*Correspondence: Giovanfrancesco.Ferrari@unibas.ch

2 University of Basel, Basel, Switzerland

Full list of author information is available at the end of the article
} 


\section{Background}

Urbanization, widespread use of malaria control measures and effective treatment in recent years have had a significant impact in reducing the prevalence of malaria in many African cities, as well as contributing to the more heterogeneous risk in malaria observed in many urban areas [1-5]. In Kinshasa, the capital of the DRC malaria has considerably decreased during the past 30 years. A survey conducted in 2011, showed a prevalence of $17 \%$ among children 6-59 months of age, and the existence of a gradient of prevalence from the centre (lower risk) to the periphery (higher risk) [6-9]. Moreover, traditionally attention has focused on high risk groups, and little attention has been put on older children and adults. Understanding the complex heterogeneity of risk factors that can contribute to increased risk of malaria in urban settings across different age groups will enable a more effective use of control measures.

In addition, many studies have shown that control of malaria can reduce the prevalence of childhood anaemia [10-14]. Anaemia, in particular due to iron deficiency, is a major public health challenge in paediatric populations in sub-Saharan Africa, and in DRC it is among the top five leading causes of years lived with disability (YLDs) (GBD 2010). In Kinshasa the current rate of anaemia $(\mathrm{Hb}<11 \mathrm{~g} / \mathrm{dl})$ among pre-school aged children was $65 \%$ in 2011 [6].

The present study investigated individual and household risk factors for Plasmodium falciparum infection and anaemia in Kinshasa in children aged 6-59 months in 25 non-rural HZs, as well as in individuals aged 5 years and older in a separate analysis in two HZs.

\section{Methods}

\section{Study area and recruitment of study participants}

This study used cross-sectional data from a survey conducted in 2011 in Kinshasa, the capital city of the DRC, which is described in detail by Ferrari et al. [6]. In summary, data collection took place from April to June 2011, before the end of the rainy season and included 2512 households selected through a multi stage sampling procedure to obtain a sample of 3342 children aged 6-59 months from 25 health zones (HZs) and 816 individuals aged 5 years or older from two HZs selected out of the 25 (Ngiri Ngiri and Selembao). The HZ represents the primary operational unit of the health system in DRC, and covers about 150,000 inhabitants. It includes a general referral hospital, health centres and lower-level health facilities. Each $\mathrm{HZ}$ is further divided in health areas. In Kinshasa malaria transmission is ensured by Anopheles gambiae s.l., and usually peaks during the long rainy season from September to May [15]. From each participant a finger-prick blood sample was collected to test for malaria by rapid diagnostic test (RDT) (SD Bioline Malaria Antigen P.f/Pan), providing an immediate on-site diagnosis. The level of haemoglobin $(\mathrm{Hb})$ was measured with a HemoCue 201 plus + photometer (Ångelholm, Sweden). Axillary temperature was measured using a digital thermometer and the individual's history of fever in the preceding 2 weeks was also recorded. A standardized electronic survey questionnaire was administered to all heads of eligible household using an HTC smartphone running with Android OS. The survey questionnaire was an adaptation of the standard malaria indicator survey questionnaire from the Roll Back Malaria Partnership (http://www.RBM.org) created with the Build component of the open data kit (ODK) software (University of Washington \& Google Foundation). Respondents were asked about demographic information of the residents, educational level, assets owned (such as television and bicycle), presence of insecticide-treated bed net (ITN) and use of ITN the night prior to the survey.

\section{Assessing risk factors of Plasmodium infection and anaemia}

The analysis was stratified according to malaria transmission intensity, based on the prevalence of malaria infection measured in 2011 among children 6-59 months [6]. The prevalence ranged from 0.7 to $46 \%$ in children aged 6-59 months. Two strata were defined at the HZ level: a prevalence of infection below $10 \%$ or a prevalence above $10 \%$. The $10 \%$ prevalence cut-off was an arbitrary selection to allow enough observations in each strata. Plasmodium infection and anaemia were assessed for their association with a number of variables. For individuals aged older than 5 years, data collection took place in only two HZs with different transmission intensities (Ngiri Ngiri, $0.8 \%$ and Selembao, $26.8 \%$ in children younger than 5 years); these data were analysed separately.

The primary outcomes of the study were the presence or absence of Plasmodium malaria as measured by rapid diagnostic test (RDT) and the anaemia test results. A child aged between 6 and 59 months was defined as anaemic if his/her $\mathrm{Hb}$ was below $11.0 \mathrm{~g} / \mathrm{dl}$. Therefore, the outcomes variables were dichotomous. Recorded explanatory variables were: age, gender, educational level of the respondent, occupation of the respondent, insecticide mosquito-net use and reported fever during the last 2 weeks and wealth index. A wealth index, calculated according to the method of Filmer et al., was constructed for each household based on ownership of household assets (having a television, a radio, etc.) and house characteristics (having electricity, drinking water, toilet type, roof and ground material) [16]. Three categories were generated to classify households ranging from the poorest to the least poor in the community. 


\section{Statistical methods}

The proportions with malaria infection and with anaemia were analysed using a logistic regression model with random effects to take account clustering by health zone and health area. All analysis were performed separately for children (6-59 months) and individuals older than 5 years since they were sampled from different HZs. The analysis was carried out using STATA version 13 (Stata Corporation College Station, TX, USA).

\section{Results}

Data collection took place in 2512 households, in the $25 \mathrm{HZs}$ that were visited. A total of 3342 children aged 6-59 months were included in the analysis, 1118 and 2224 in the low and high transmission settings, respectively. A similar number of males (50\%) and females were included; the median age was 30 months $(90 \%$ central range 9-55). Table 1 shows the number of children examined, by $\mathrm{HZ}$ and by transmission strata. For individuals above 5 years of age, data collection took place in two HZs only and included 816 individuals, of which $34 \%$ were males and the median age was 22 years (90\% central range 6-62).

\section{Risk factors for Plasmodium infection in children aged 6-59 months (25 HZs)}

The risk factors for Plasmodium infections in children 6-59 months are shown in Table 2. There was an increase in the proportion with malaria infection with age in both transmission strata. The greatest risk was in children 48-59 months: an odds ratio (OR) of 5.86 [95 \% confidence interval (CI) 1.62-21.17] for the 36-47 months group and an OR of 15.53 (95\% CI 4.26-56.64) for the 48-59 months group, compared to the youngest age group. The effect was also seen in higher transmission strata, although the OR was lower: an OR of 1.73 (95\% CI 1.36-2.20) for the 36-47 months group and an OR of 2.54 (95\% CI 1.93-3.35) for the 48-59 months group compared to the youngest age group. The interaction between age and transmission intensity was significant $(\mathrm{p}=0.009)$.

Treated net use was found to significantly lower malaria infection risk in the higher transmission strata with $38 \%$ protection (OR $=0.62,95 \%$ CI $0.50-0.77)$, however the effect was not significant in the lower transmission strata. Children who reported fever in the last 2 weeks had a significantly elevated risk of malaria infection in both strata.

Higher education levels showed a trend towards being protective in both transmission settings (Table 2). However there was no evidence of an association with the occupation of the respondent. Finally, children living in the wealthiest tertile were significantly less likely to have a malaria infection compared to the children from the poorest tertile in strata of high transmission $(\mathrm{OR}=0.27$,
$95 \%$ CI $0.20-0.38, \mathrm{p}<0.001)$. No evidence was found in the HZs with less than $10 \%$ prevalence $(\mathrm{OR}=0.82,95 \%$ CI $0.31-2.13, p=0.83$ ), however the interaction between socioeconomic status and transmission was not significant $(\mathrm{p}=0.14)$.

\section{Risk factors for Plasmodium infection in individuals older than 5 years $(2 \mathrm{HZs})$}

The risk factors for Plasmodium infection in individuals aged older than 5 years are shown in Table 3. The association between age and malaria infection was strong. The highest prevalence was observed in the 15-19 years age group in the low transmission HZ of Ngiri Ngiri with an OR of 7.11 (95\% CI 1.17-43.05) compared to the 5-9 years-old. In the higher transmission HZ of Selembao however, ORs were lower and more homogeneously distributed across all age groups, compared to the 5-9 years-old group which showed the highest prevalence. The interaction between age and transmission intensity however was not significant $(\mathrm{p}=0.11)$.

ITN use was not found to significantly lower the prevalence of malaria infection, although the estimates were in the direction of being protective. Individuals aged 5 years and older who reported fever in the last 2 weeks had an elevated risk of having malaria infection in both sites, and the association was stronger for the lower transmission: $\mathrm{OR}=38.71$ (95 \% CI 11.08-135.23), and OR $=2.05$ (95\% CI 1.07-3.95) in Selembao, with a highly significant interaction term $(\mathrm{p}<0.0001)$. There was no evidence of an effect of higher education levels, occupation of the respondent or socio-economic status.

\section{Risk factors for anaemia in children aged 6-59 months (25 HZs)}

The risk of having anaemia was found to decline progressively with increasing age (Table 4 ) in both low and high transmission strata $(p<0.001)$. Although there was no evidence that malaria infection increased the risk of having anaemia in the low transmission strata $(\mathrm{OR}=2.01$, $95 \%$ CI 0.89-4.51), this effect was significant in the higher transmission strata (OR $=3.40,95 \%$ CI 2.60 4.44). There was no evidence that reported ITN use was protective for the anaemia status in either strata. There was also no evidence of an association with fever, nor with education or occupation. Belonging to the wealthiest tertile was borderline significantly associated with the risk of having anaemia in both low transmission (OR $0.68,95 \%$ CI 0.47-0.99) and high transmission strata.

\section{ITN use}

There were some age-specific differences in ITN usage (Fig. 1), with highest use in younger children $(\mathrm{p}=0.006)$ in the low transmission strata. In areas of high 


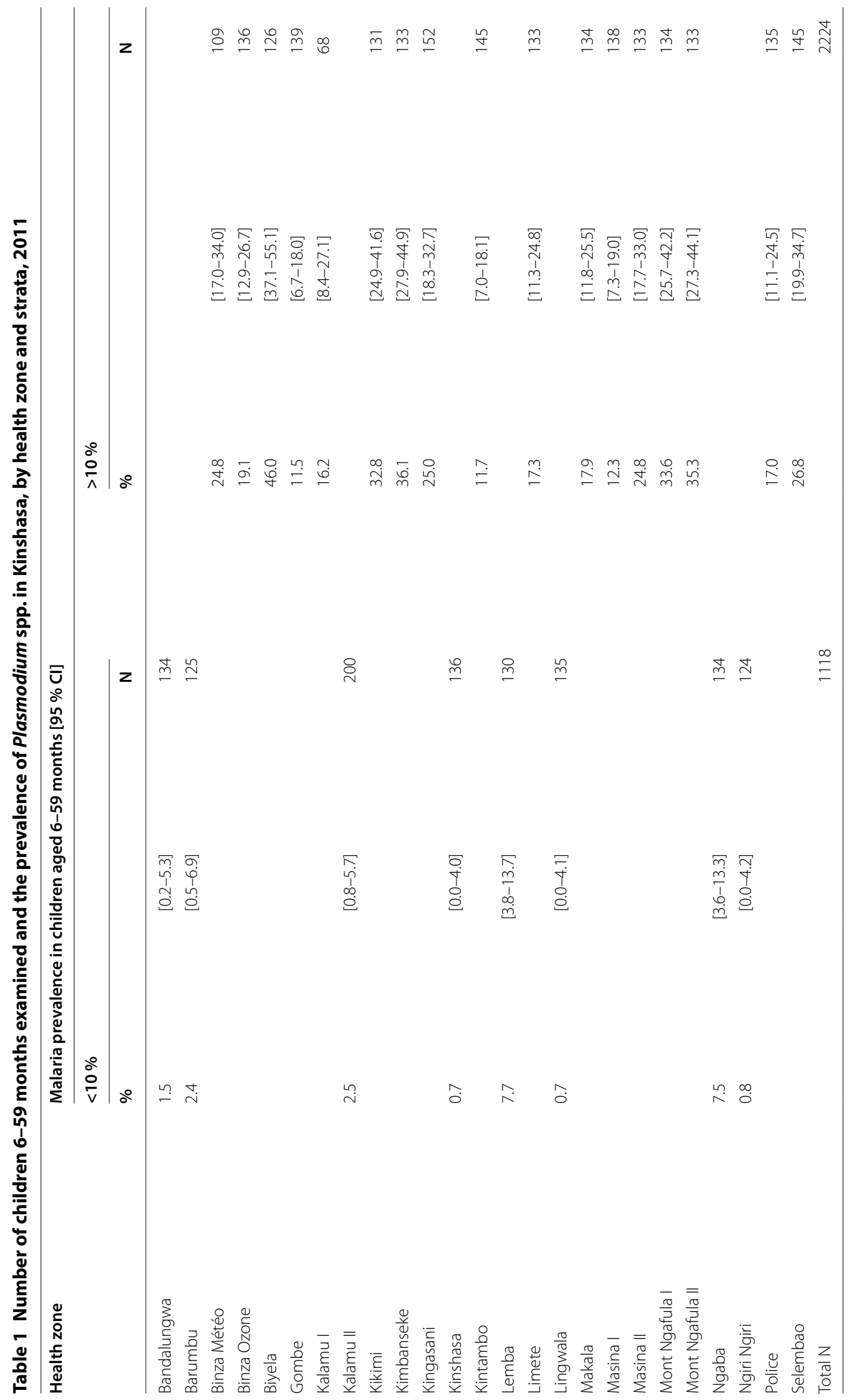




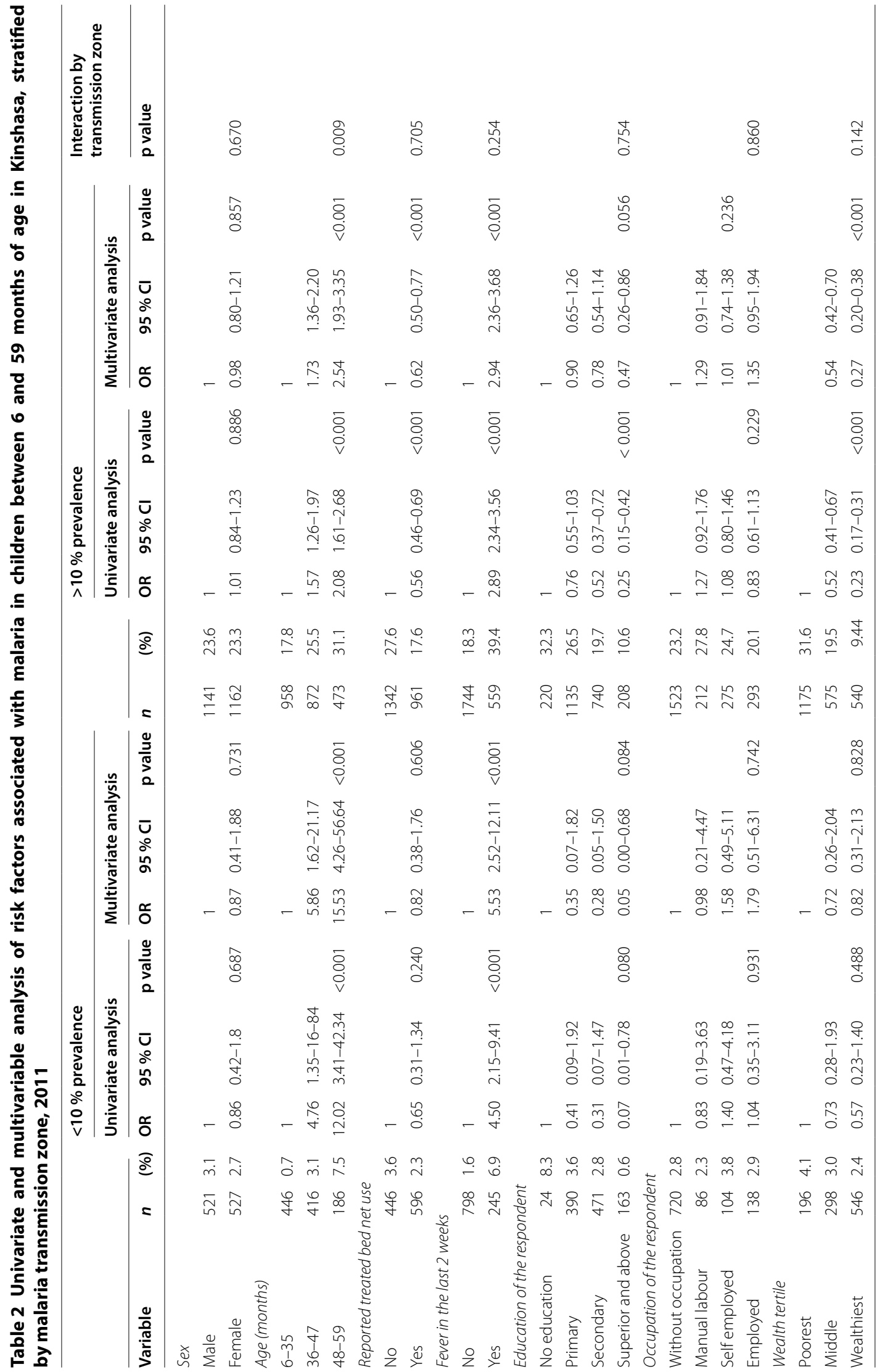




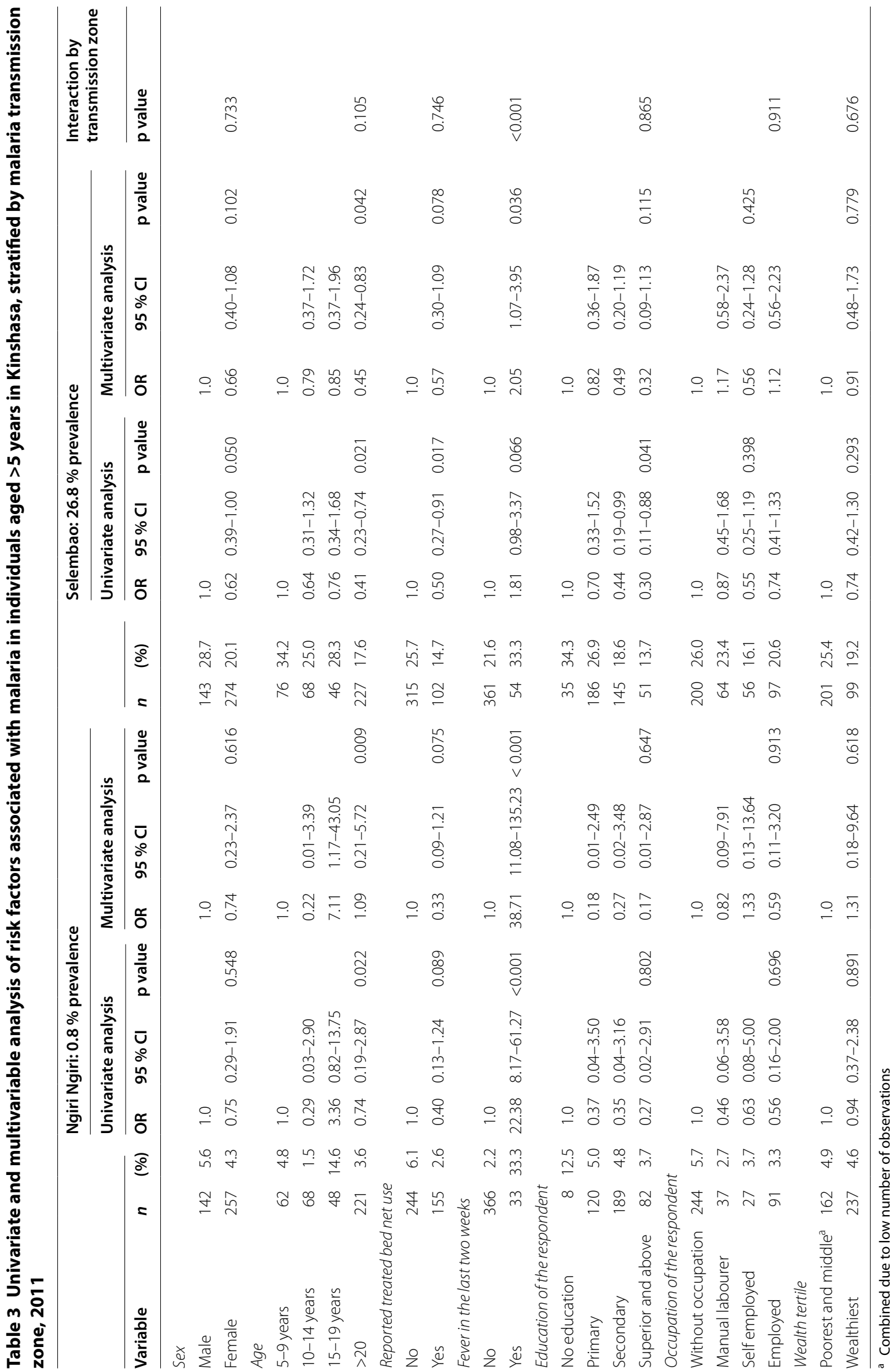




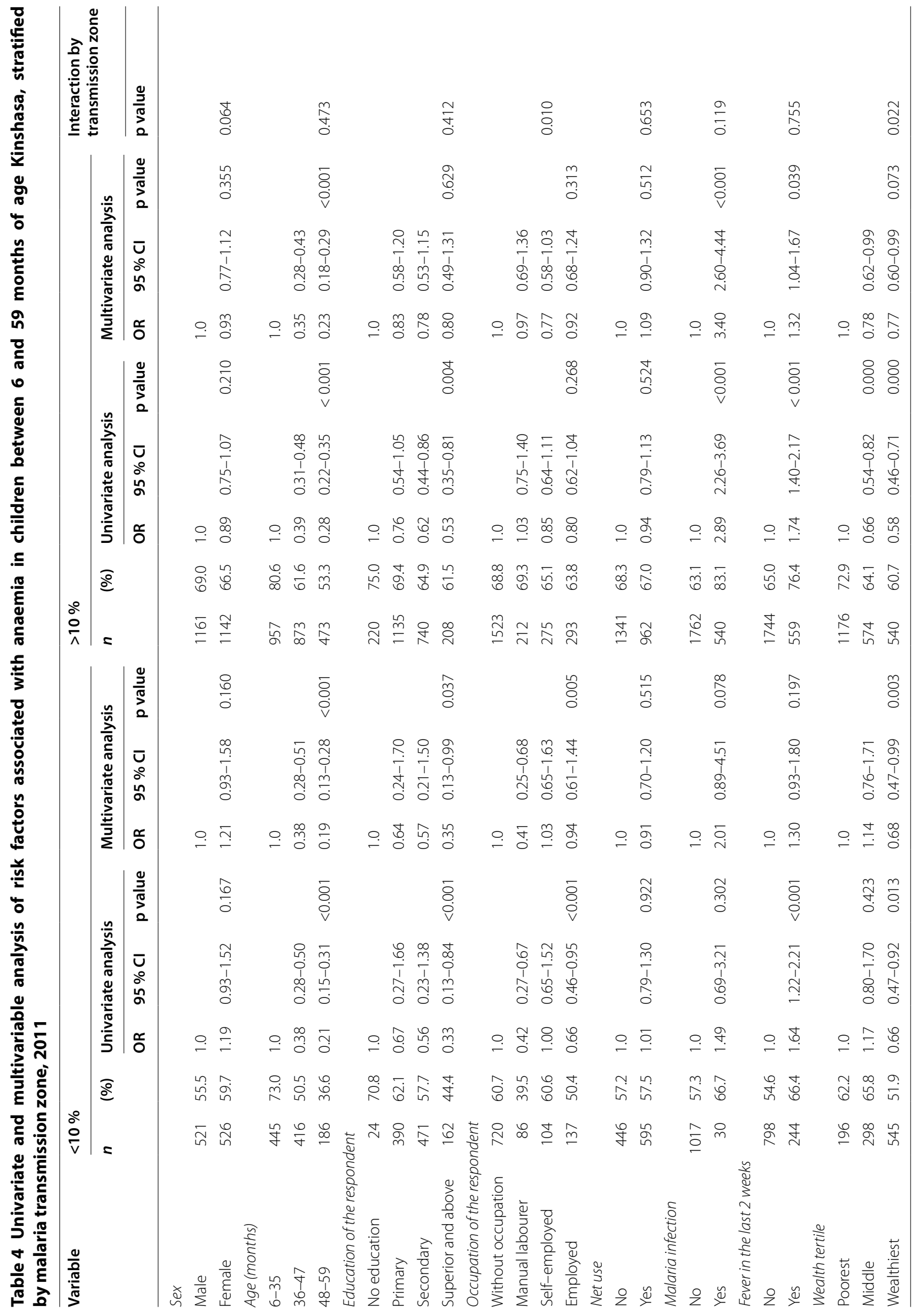


transmission, ITN usage although lower appeared more homogeneously distributed across age groups (Fig. 1). No significant differences in the utilization were found among individuals age more than 5 years, in both low and high transmission strata (Fig. 1).

\section{Discussion}

The identification of risk factors for malaria infection and anaemia, provides information on the local malaria epidemiology and has the potential to lead to a more effective and targeted use of malaria control measures. This study presents the results of an analysis of the association of a number of variables that alone or in combination could affect the risk of acquiring Plasmodium infection and anaemia, in a city with diverse malaria transmission patterns. The key results from this analysis are the association between malaria infection and age, with older age groups being exposed to higher risk of malaria in low transmission settings and a lower and more homogeneous risk across all age groups in high transmission settings. Shift in the age of peak prevalence towards the older groups has been described for malaria and other infectious diseases and is consistent with exposure-related acquired immunity $[17,18]$. In zones of low transmission, children are less exposed to infective malaria, hence delaying the age of the first infection and the acquisition of immunity [19]. Clearly, in Kinshasa the risk of Plasmodium infection seems to occur later in childhood, which is consistent with areas of rather low levels of transmission. The prevalence rates by $\mathrm{HZ}$ shown in Table 1 (range: 0.7-46.0), with most HZs below $30 \%$ confirm that Kinshasa overall has a moderate endemicity level. Recent school surveys done in Kimbanseke, a relatively high prevalence $\mathrm{HZ}$ southeast of Kinshasa, found similar results, showing children aged 10-13 at higher risk of malaria and a high prevalence of asymptomatic infections [20].

The relationship of ITN use by the different age groups could also influence the age pattern of risk that was observed: children in the youngest age group, 6-35 months, were significantly more likely to sleep under an ITN in the low transmission setting, whereas utilization was similar among age groups in high transmission setting. A similar shift in age of peak of prevalence towards the older children has been observed elsewhere with an increase in net coverage [21]. Only $44 \%$ of children 6-59 months used an ITN the night preceding the survey, which is still far from universal coverage. In individuals $>5$ years, overall ITN use was even lower, with only 38 and $23.3 \%$ using an ITN in Ngiri
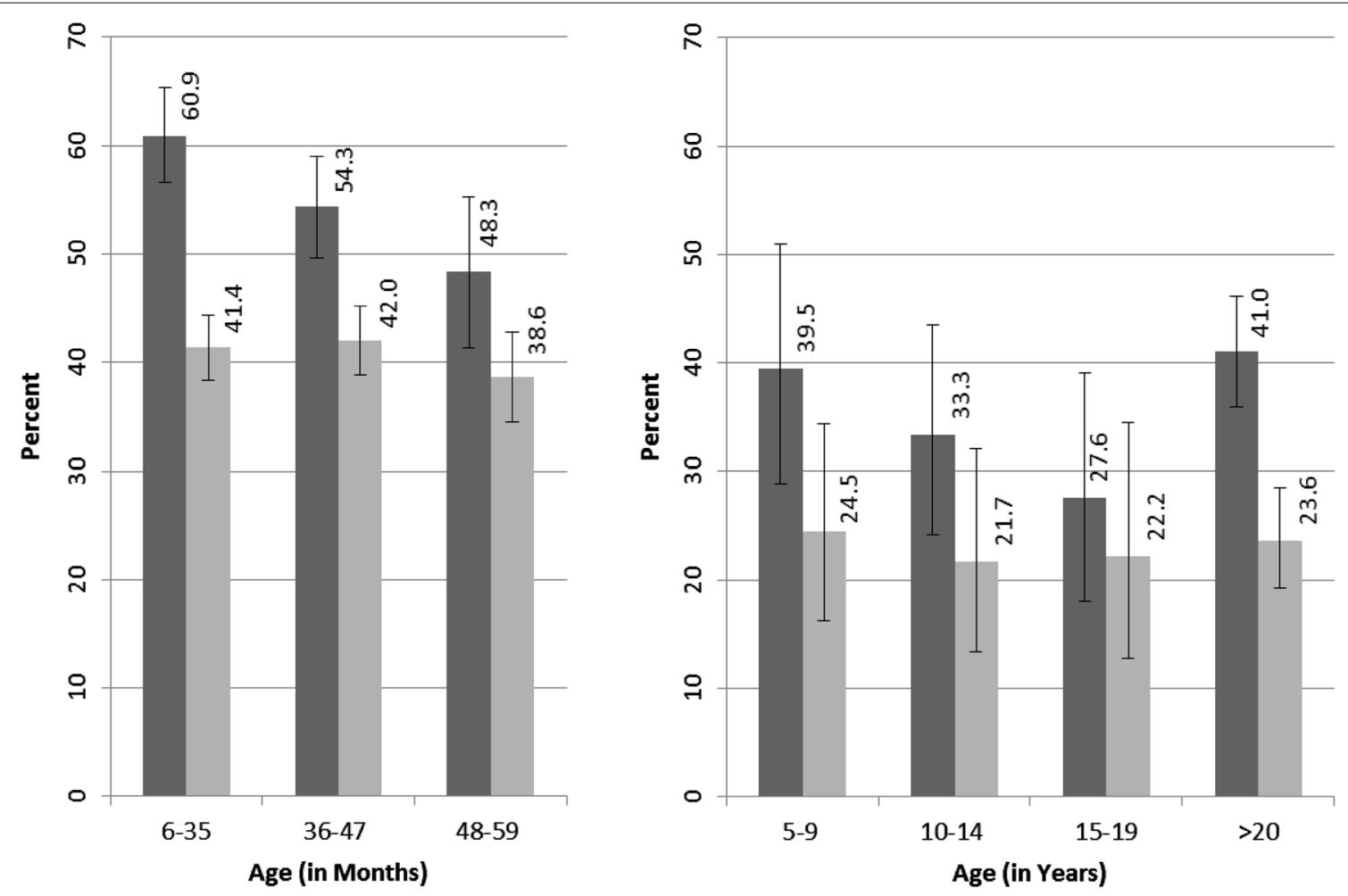

Fig. 1 Left panel ITN usage among children 6-59 months by transmission intensity. Right panel ITN usage among individuals older than 5 years in Ngiri Ngiri (0.8\%) and Selembao (26.8\%) in Kinshasa, 2011. Light grey bars represent proportions of reported ITN usage in high transmission strata, and dark grey bars in low transmission strata. Error bars indicate $95 \% \mathrm{Cl}$ 
Ngiri and Selembao, respectively. In addition, less than $58 \%$ of HHs owned enough ITNs to cover all household members in 2011. These low values are of concern.

Higher malaria prevalence in older children has also been attributed to increased use of anti-malarials in early childhood [22]. In case of fever in Kinshasa, it is common practice by the caregivers to initially treat their child at home (54\% of the cases) although only $4.3 \%$ of the children treated for fever receive a recommended combination therapy containing artemisinin (unpublished data).

Results indicated that sleeping under an ITN the previous night reduced the risk of Plasmodium infection by $38 \%$ (OR 0.62, $95 \%$ CI 0.50-0.77) among children 6-59 months of age in areas of high transmission, consistent with the vast body of evidence supporting the efficacy and effectiveness of ITN in protecting against malaria [11]. In low transmission areas, however, there was no evidence of such an association, presumably because the overall risk of infection was lower.

Reported history of fever was associated with malaria infection overall. There was evidence of a difference in this association with transmission level among individuals aged older than 5 years $(\mathrm{p}<0.001)$. The weaker association of reported fever with malaria in areas of high transmission could be explained by differences in the levels of acquired immunity.

The data confirm that anaemia is frequent in urban Kinshasa, with $65 \%$ prevalence among children 6-59 months, $30 \%$ moderate $(7.0-9.9 \mathrm{~g} / \mathrm{dl})$ and $1.9 \%$ severe $(<7.0 \mathrm{~g} / \mathrm{dl})$. ITN use in Kinshasa did not appear to be associated with benefits in lower anaemia risk, contrary to what has been documented in other settings [1014]. These findings are consistent with a Kenyan study that found only a small difference in prevalence of anaemia between villages with and without ITNs [23]. Anaemia has many causes in addition to malaria [nutrition [24], soil transmitted infections ( $\mathrm{STH}$ ) and schistosomes], and in Kinshasa these are likely to also be contributors to this morbidity. A recent study revealed a high prevalence of STH infections among primary school children in Kinshasa (32.8\%) [20]. Nevertheless, the estimated odds of anaemia in zones of high transmission were 3.5 times (95\% CI 2.70-4.62) higher in malaria infected children.

In this study, the risk of anaemia was shown to decrease with increasing age in both low and high transmission strata. These results are consistent with studies conducted in West Africa, showing a significant reduction in the mean haemoglobin level in children aged $2-5$ years compared to children aged 1-2 [25].

This study also showed differences in the effect in malaria risk or anaemia risk by socioeconomic status, consistent with previous studies carried out in
sub-Saharan Africa [19, 26-28], and as documented in a multi-country analysis of DHS data [29].

This study however has some limitations. Foremost, the analysis draws on cross-sectional data; hence the causal nature of associations should be viewed with a certain caution. A second most important limitation of the study relates to the smaller sample size for individuals over 5 years compared to that of children 6-59 months, limiting the authors' ability to potentially detect important differences and interactions between risk factors and transmission. Furthermore, the low proportion of males for this survey (34\%) may have triggered a gender-response bias, with consequences on the prevalence and associations found. The direction and magnitude of a possible bias remain unknown. Lastly, RDTs are limited in sensitivity to detect low density parasitaemia and their use may have led to an underestimation of the true proportion of people infected with $P$. falciparum. The underestimation may have differed with acquired immunity affecting the age pattern [30-33].

\section{Conclusions}

For the most part, there was no evidence of an interaction between malaria infections and the risk factors with transmission intensity; however the associations with age and with fever in the last 2 weeks did vary significantly. The results also show that school-aged children are the least protected with ITN, across the different transmission settings, hence representing an important reservoir for infection. The observation of a shift in the peak age of risk for malaria to older groups is consistent with areas of low transmission and highlights the need for a more equal distribution of ITN in Kinshasa to target all age groups and not only the traditional high-risk group of young children.

\section{Abbreviations \\ HA: health area; $\mathrm{HZ}$ : health zone; ITN: insecticide-treated net; RDT: rapid diagnostic test.}

\section{Authors' contributions}

$\mathrm{CL}$ and ATK conceived the study. GF, HMN and SS conducted the field work and provided supervision throughout the duration of the study. GF and HMN contributed to the management, analysis and interpretation of the data. AR provided assistance with statistical analysis. GF drafted the manuscript. All authors contributed to the editing of the manuscript. All authors read and approved the final manuscript.

\section{Author details \\ ${ }^{1}$ Swiss Tropical and Public Health Institute, P.O. Box, 4002, Basel, Switzerland. ${ }^{2}$ University of Basel, Basel, Switzerland. ${ }^{3}$ Kinshasa School of Public Health, Kinshasa, Democratic Republic of the Congo.}

\section{Acknowledgements}

The authors thank the FEVERKIN staff for the efforts in the collection of data and for the excellent work, as well as all patients and parents for their 
collaboration. We are grateful to Ministry of Health authorities and to the National Malaria Control Programme staff for support.

\section{Competing interests}

The authors declare that they have no competing interests.

\section{Availability of data and materials}

The datasets supporting the conclusions of this article are available from the corresponding author on request.

\section{Consent for publication}

Written informed consent to publication was obtained from each participant.

\section{Ethics approval and consent to participate}

Ethical approval of the study was obtained from the ethics committee of the Kinshasa School of Public Health University of Kinshasa, in DRC, as well as the ethical committee in Basel (Ethikkommission beider Basel, Basel-Stadt). Individual written informed consent was obtained by parents or guardian on behalf of their children (until the age of 10) or by the adults study participants themselves. In addition, assent was obtained from children over 10 years of age. Every precaution to minimize the risk of infection during blood sampling was taken. All patients who tested positive for malaria by RDT were treated for free by the nationally recommended therapy combination with artemisinin, artesunate plus amodiaquine (ASAQ), previously placed at the health centre of reference of the corresponding health area.

\section{Funding}

The study received financial support from the UBS Optimus Foundation.

Received: 5 February 2016 Accepted: 1 July 2016

Published online: 15 July 2016

\section{References}

1. Keiser J, Utzinger J, Caldas de Castro M, Smith TA, Tanner M, Singer BH. Urbanization in sub-saharan Africa and implication for malaria control. Am J Trop Med Hyg. 2004;71:118-27.

2. Matthys B, Vounatsou P, Raso G, Tschannen AB, Becket EG, Gosoniu L, et al. Urban farming and malaria risk factors in a medium-sized town in Cote d'Ivoire. Am JTrop Med Hyg. 2006;75:1223-31.

3. Staedke SG, Nottingham EW, Cox J, Kamya MR, Rosenthal PJ, Dorsey G. Proximity to mosquito breeding sites as a risk factor for clinical malaria episodes in an urban cohort of Ugandan children. Am J Trop Med Hyg. 2003;69:244-6.

4. Wang SJ, Lengeler C, Smith TA, Vounatsou P, Cisse G, Diallo DA, et al. Rapid urban malaria appraisal (RUMA) in sub-Saharan Africa. Malar J. 2005:4:40.

5. Caldas de Castro M, Yamagata Y, Mtasiwa D, Tanner M, Utzinger J, Keiser J, et al. Integrated urban malaria control: a case study in Dar es Salaam. Tanzania. Am J Trop Med Hyg. 2004;71:103-17.

6. Ferrari G, Ntuku HM, Schmidlin S, Diboulo E, Tshefu AK, Lengeler C. A malaria risk map of Kinshasa, Democratic Republic of Congo. Malar J. 2016;15:27.

7. Kazadi W, Sexton JD, Bigonsa M, W'Okanga B, Way M. Malaria in primary school children and infants in Kinshasa, Democratic Republic of the Congo: surveys from the 1980s and 2000. Am J Trop Med Hyg. 2004;71:97-102.

8. Mulumba MP, Wery M, Ngimbi NN, Paluku K, Van der Stuyft P, De Muynck A. [Childhood malaria in Kinshasa (Zaire). Influence of seasons, age, environment, and family social conditions](in French). Trop (Mars). 1990;50:53-64.

9. Ngimbi NP, Beckers A, Wery M. [Survey of the epidemiological status of malaria in Kinshasa (Republic of Zaire) in 1980](in French). Ann Soc Belg Med Trop. 1982;62:121-37.

10. Korenromp EL, Armstrong-Schellenberg JR, Williams BG, Nahlen BL, Snow RW. Impact of malaria control on childhood anaemia in Africa-a quantitative review. Trop Med Int Health. 2004;9:1050-65.

11. Lengeler $C$. Insecticide-treated bed nets and curtains for preventing malaria. Cochrane Database Syst Rev. 2004;2:CD000363.
12. Premji Z, Hamisi Y, Shiff C, Minjas J, Lubega P, Makwaya C. Anaemia and Plasmodium falciparum infections among young children in an holoendemic area, Bagamoyo, Tanzania. Acta Trop. 1995;59:55-64.

13. Premji Z, Lubega P, Hamisi Y, McHopa E, Minjas J, Checkley W, et al. Changes in malaria associated morbidity in children using insecticide treated mosquito nets in the Bagamoyo district of coastal Tanzania. Trop Med Parasitol. 1995;46:147-53.

14. Mathanga DP, Campbell CH Jr, Vanden Eng J, Wolkon A, Bronzan RN, Malenga GJ, et al. Comparison of anaemia and parasitaemia as indicators of malaria control in household and EPI-health facility surveys in Malawi. Malar J. 2010;9:107.

15. Coene J. Malaria in urban and rural Kinshasa: the entomological input. Med Vet Entomol. 1993;7:127-37.

16. Filmer D, Pritchett LH. Estimating wealth effects without expenditure data-or tears: an application to educational enrollments in states of India. Demography. 2001;38:115-32.

17. Woolhouse ME. Patterns in parasite epidemiology: the peak shift. Parasitol Today. 1998;14:428-34.

18. Snow RW, Marsh K. The consequences of reducing transmission of Plasmodium falciparum in Africa. Adv Parasitol. 2002;52:235-64.

19. Winskill P, Rowland M, Mtove G, Malima RC, Kirby MJ. Malaria risk factors in north-east Tanzania. Malar J. 2011;10:98.

20. Matangila JR, Doua JY, Linsuke S, Madinga J, Inocencio da Luz R, Van Geertruyden JP, et al. Malaria, schistosomiasis and soil transmitted helminth burden and their correlation with anemia in children attending primary schools in Kinshasa, Democratic Republic of Congo. PLoS One. 2014;9:e110789.

21. Smith T, Hii JL, Genton B, Muller I, Booth M, Gibson N, et al. Associations of peak shifts in age-prevalence for human malarias with bednet coverage. Trans R Soc Trop Med Hyg. 2001;95:1-6.

22. Trape JF. Malaria and urbanization in central Africa: the example of Brazzaville. Part IV. Parasitological and serological surveys in urban and surrounding rural areas. Trans R Soc Trop Med Hyg. 1987;81 (Suppl 2):26-33.

23. Desai MR, Terlouw DJ, Kwena AM, Phillips-Howard PA, Kariuki SK, Wannemuehler KA, et al. Factors associated with hemoglobin concentrations in pre-school children in Western Kenya: cross-sectional studies. Am J Trop Med Hyg. 2005;72:47-59.

24. UNICEF. Multiple Indicator Cluster Survey (MICS), Democratic Republic of Congo, Preliminary Findings. 2010.

25. Soares Magalhaes RJ, Clements AC. Spatial heterogeneity of haemoglobin concentration in preschool-age children in sub-Saharan Africa. Bull World Health Organ. 2011;89:459-68.

26. De Beaudrap P, Nabasumba C, Grandesso F, Turyakira E, Schramm B, Boum $Y$, et al. Heterogeneous decrease in malaria prevalence in children over a six-year period in south-western Uganda. Malar J. 2011;10:132.

27. Gahutu JB, Steininger C, Shyirambere C, Zeile I, Cwinya-Ay N, Danquah I, et al. Prevalence and risk factors of malaria among children in southern highland Rwanda. Malar J. 2011;10:134.

28. Ayele DG, Zewotir TT, Mwambi HG. Prevalence and risk factors of malaria in Ethiopia. Malar J. 2012;11:195.

29. Balarajan Y, Ramakrishnan U, Ozaltin E, Shankar AH, Subramanian SV. Anaemia in low-income and middle-income countries. Lancet. 2011;378:2123-35.

30. Harris I, Sharrock WW, Bain LM, Gray KA, Bobogare A, Boaz L, et al. A large proportion of asymptomatic Plasmodium infections with low and sub-microscopic parasite densities in the low transmission setting of Temotu Province, Solomon Islands: challenges for malaria diagnostics in an elimination setting. Malar J. 2010;9:254.

31. Alves FP, Durlacher RR, Menezes MJ, Krieger H, Silva LH, Camargo EP. High prevalence of asymptomatic Plasmodium vivax and Plasmodium falciparum infections in native Amazonian populations. Am J Trop Med Hyg. 2002;66:641-8.

32. Nicastri E, Bevilacqua N, Sane Schepisi M, Paglia MG, Meschi S, Ame SM, et al. Accuracy of malaria diagnosis by microscopy, rapid diagnostic test, and PCR methods and evidence of antimalarial overprescription in nonsevere febrile patients in two Tanzanian hospitals. Am J Trop Med Hyg. 2009:80:712-7.

33. Wu L, van den Hoogen LL, Slater H, Walker PG, Ghani AC, Drakeley CJ, Okell LC. Comparison of diagnostics for the detection of asymptomatic Plasmodium falciparum infections to inform control and elimination strategies. Nature. 2015;528:S86-93. 